\title{
Magnitude of Diabetes Comorbidity among People Living with HIV: A Systematic Review
}

\author{
Tilahun NigatuHaregu ${ }^{1, *}$, B rian Olde nburg ${ }^{1}$, Geoffrey Se ts we $^{2}, \mathrm{Julian}^{\text {Elliott }}{ }^{3}$ \\ ${ }^{1}$ Department of Epidemiology and Preventive Medicine, Monash University, Australia \\ ${ }^{2}$ School of Health Sciences, Monash University, South Africa \\ ${ }^{3}$ Infectious disease unit, Alfred Hospital, Melbourne, Australia
}

\begin{abstract}
Although the clinical relationship between HIV/AIDS and Diabetes is well established, there is a little summarized evidence about the magnitude ofDiabetescomorbidity a mong people living with HIV.The aim of this study was to summarizeevidence on the incidence and prevalence of Diabetes comorbid ityamong people living with HIV.A systematic review of the incidence and prevalence of Diabetes comorbidity among people living with HIV was conducted. Studies reporting incidence and/orprevalence of diabetes among people living with HIV were retrieved from Embase and Medline databases. Selection of the studies was based on both relevance and quality.Study and outcome characteristics, were extracted using a standardized checklist. Resulted were presented using narrative and graphic summaries. PRISMA checklist was used as a guide for reporting of the review results. A total of 12 studies met the inclusion and quality. In total of 43,296 people liv ing with HIV, 1,144 incident cases of diabetes were identified over 174,574 person-years. The incidence rates of diabetes comorbid ity as reported by these studies ranged from 5.72 to 23.8 per 1000 person-years. Similarly, a total of 1,724 prevalent cases of diabetes were identified among 41,068 people living with HIV. The reported prevalence rates ranged from $2.85 \%$ to $14.9 \%$. High level of variability in the reported incidence and prevalence rates of diabetes comorbidity was observed. Generally, the findings are not in favour of a significantly increased magnitude of diabetes comorbidity in HIV.
\end{abstract}

Keywords HIV-Diabetes Comorbidity, Magnitude, Prevalence, Incidence

\section{Introduction}

While still having adisproportionate burden of infectious diseases, most low and middle income countries are now facing a rapidly emerging burden of Noncommunicable diseases. This epidemiological overlap is expected to result in higher rates of comorbidity[1]. It is generally well understood that infectious diseases increase the risk of non-communicable diseases. Conversely, non-communicable diseases also predispose people to infectious diseases[2]. Besides, some infectious diseases increase the risk of getting other infectious diseases resulting in higher coinfection rates [3]. This also holds true for Noncommun icable diseases. Hence, the interrelationships between diseases are so complex that comorbidities exist in a significant magnitude and are worth considering.

There is no standard definition of comorbidity. It may refer to diseases or disorders that exist together with an index disease; or it may refer to the co-occurrence of two or more diseases in an individual. Measurement of comorbidity is currently gettingmore attention in medical research. Data

* Corresponding author:

tilahunn@gmail.com(Tilahun NigatuHaregu)

Published online at http://journal.sapub.org/diabetes

Copyright (C) 2012 Scientific \& Academic Publishing. All Rights Reserved sources that are available to measure comorbidity include medical records, patient self-report, clinical judgement and administrative databases. Comorbidity can take four major forms in statistical analysis: a confounder, an effect modifier, an exposure and an outcome. Most comorbidity studies involve the development andapplication of comorbidity indices to understand the effect of comorbidities on defined health outcomes [4].

Diabetes comorbidity in HIV infection, the occurrence of Diabetes in an individual living with HIV, is receiv ingmo re concernin line with the emergence of diabetes as a major disease of public health importance in high HIV prevalent countries. Low and middle income countries which already have high magnitude of HIV are expected to share high burden of Diabetes comorbidity due to the increase in the incidence of Diabetes in these settings [5].

Though more is known about the clinical relationship between HIV and Diabetes and the factors associated with that relationship, little is known about the epidemiology of their comorbidity. W ith the emergence of double mortality burden in low and middle income countries, better understanding of the epidemiology of HIV-Diabetes comorbidity is essential[6,7].Data on the incidence and prevalence of Diabetes among people with HIV are availab le from different studies in different parts of the world. There are also several studies that have reported the effect of 
antiretroviral treatment on the risk of developing Diabetes among people living with HIV. However, to the knowledge of the investigators, there is no research effort that summarizes altogether the incidence and prevalence of Diabetes comorbid ityin HIV infection.

Therefore, the aim of this study was to review and summarizethe incidence and prevalenceof Diabetes comorbidity among people living with HIV andthereby to discuss their imp lications on possible integration of HIV and Diabetes programs.

\section{Methods}

\subsection{Search Strategy and Study Selection}

We searched studies reportingtheincidence and/or prevalence of Diabetes comorbidity among people living with HIV from Embase and Medlineelectronic databases (March 2012). We used the key words of "HIV and Diabetesand incidence" and "HIV and Diabetes and prevalence"in Embase search. We used the MeSH terms "HIV infection" and "Diabetes mellitus" in Medline. The titles and abstracts of the identified studies were screened for eligibility. The references of the identified reviews were consulted without restriction to identify additional studies.

We reviewed the full texts of all the retrieved articles for assessment ofelig ibility. The inclusion of the articles in to the review was based on the following eligibility criteria: 1) Studies reporting primary data (reviews were excluded); 2) studies reporting the prevalence and/or incidence of diabetes 3) studies conducted entirely on people living with HIV; and 4) Studies reporting explicit study design, sample size, follow up period, and outcome ascertainment.

For practical reasons, one author $(\mathrm{TN})$ conducted the screening and the eligibility assessment and the other authorshave re-checked the screening and the eligibility assessment process and outputs.

A total of 22 studies met the eligibility criteria. Nine of these have reported incidence of diabetes in people living with HIV. Sixteen studies have reported prevalence of diabetes comorbidity among people living with HIV. Three studies have reported both on the incidence and prevalence of diabetes comorbidity among people living with HIV.

\subsection{Quality Assess ment}

Risk of bias in the incidence and prevalence estimates was assessed by using five major criteria (adequacy of sample size, patient selection methods used, diabetes diagnosis methods used, quality assurance schemes applied and analysis conducted to account for the effects of possible confounding factors). Each of these criteria were scored out of four points ( $4=$ Acceptable, $3=$ Adequate, $2=$ inadequate, $1=$ not acceptable) by two reviewers. Ten artic les with scores less than half of the total score $(<10$ points $)$ were excluded.
The main reason for exclusion was inadequate sample size used for the estimation of incidence and prevalence. The final 12 studies were included in this review.

\subsection{Data Extraction}

We have extracted two categories of data from the identified studies using pre-testedchecklists. The first set of variables extracted were study characteristics including name of first author, year of publication, study design, follow up period (if applicable), sample size, and study population characteristics (age, sex, Body Mass Index (BMI), treatment status). The second set of variables was outcome characteristics which includebaseline prevalence (if applicable), incidence rates, prevalence rates and values for measures of association.

Two reviewers independently extracted data from the selected studies. Discrepancies were settled through consensus and by rechecking the individual study reports.

\subsection{Synthesis Methods}

For the included studies confidence intervals for the incidence and prevalence rates were recalculated using poison exact method in using Stata 12. As Meta-analysis of the incidence and prevalence rates was precluded by heterogeneity of the studies, we have summarized the findings of the review using narrative and graphic summaries.

\section{Results}

\subsection{Study Characteristics}

Of the 12 studies included in this systematic review, five have reported incidence and seven have reported on the prevalence (one study has reported both prevalence and incidence rates of diabetes). The search and selection of the studies is shown using the PRISMA flow diagram (Figure 1).

All the included studies have sample size of greater than 500 and were published between 2005 and 2012. Detailed study characteristics and outcome characteristics including the prevalence and incidence of diabetes (with 95\% confidence interval)from the included studies are presented in Table 1.

\subsection{Incidence of Diabetes Comor bi dity}

In the five incidencestudies, a total of 43,296 people liv ing with HIV were followed over a period of 174,574 person-years. During this follow up period a total of 1,144 incident cases of diabetes were identified. The incidence rates of diabetes comorbidity as reported by these studies ranged from 5.72 to 23.8 per 1000 person-years. The review results of individual incidence studies are described as follows. 


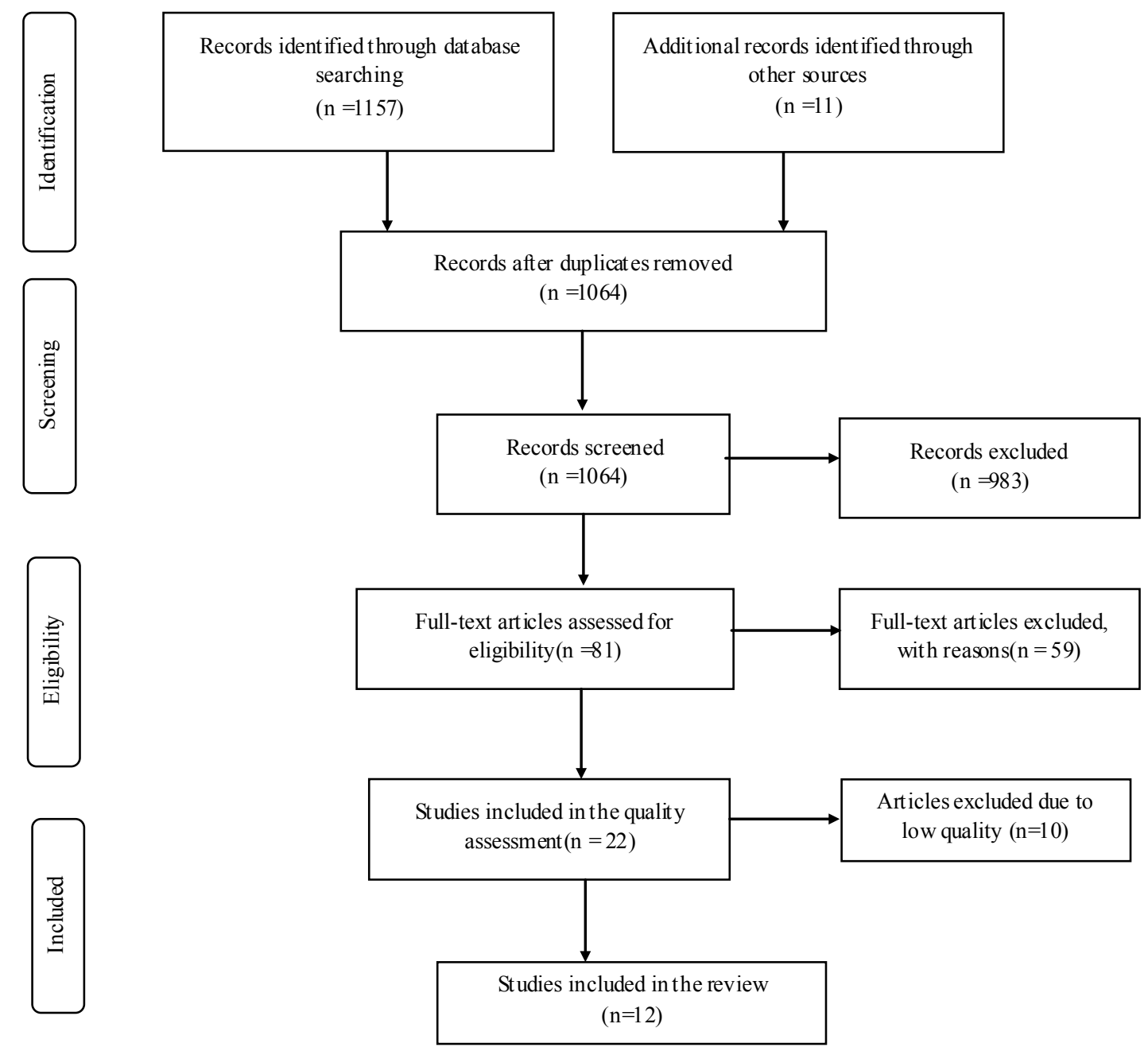

Figure 1. PRISMA Flow Diagram

Table 1. Study and Outcome Characteristics reviewed in the study

\begin{tabular}{|c|c|c|c|c|c|c|c|}
\hline First Author & $\begin{array}{c}\text { Year of } \\
\text { publication }\end{array}$ & Study Design & Outcome & $\begin{array}{c}\text { Sample } \\
\text { size }\end{array}$ & $\begin{array}{c}\text { Diabetes } \\
\text { cases }\end{array}$ & $\begin{array}{l}\text { Person } \\
\text { Years } \\
\end{array}$ & $\begin{array}{l}\text { Incidence (per } 1000 \text { PYs) or } \\
\text { Prevalence (\%) and } 95 \% \text { CI }\end{array}$ \\
\hline De Wit $S$, et $\mathrm{al}^{8}$ & 2008 & Cohort (prosp) & Incidence & 33389 & 744 & 130,151 & $5.72[5.31-6.14]$ \\
\hline Ledergerber $\mathrm{B}$, et $\mathrm{al}^{9}$ & 2007 & Cohort (prosp) & Incidence & 6513 & 123 & 27,798 & $4.42[3.67-5.28]$ \\
\hline Tien PC, et $\mathrm{al}^{10}$ & 2007 & Cohort (prosp) & Incidence & 1524 & 116 & 4,962 & $23.38[19.32-28.04]$ \\
\hline Capeau J, et $\mathrm{al}^{11}$ & 2012 & Cohort (prosp) & Incidence & 1046 & 111 & 7,846 & $14.15[11.64-17.03]$ \\
\hline Lo Y-C, et al ${ }^{12}$ & 2006 & Case-control & Incidence & 824 & 50 & 3817 & $13.10[9.72-17.27]$ \\
\hline De Wit $S$, et $\mathrm{al}^{8}$ & 2008 & Cohort(index) & Prevalence & 33389 & 952 & NA & $2.85[2.67-3.04]$ \\
\hline Butt AA, et $\mathrm{al}^{13}$ & 2009 & Cohort(index) & Prevalence & 3227 & 481 & NA & $14.9[13.69-16.18]$ \\
\hline Brar I, et $\mathrm{al}^{14}$ & 2007 & Cross-sectional & Prevalence & 1876 & 90 & NA & $4.8[3.87-5.86]$ \\
\hline Irene $\mathrm{H}$, et $\mathrm{al}^{15}$ & 2009 & Cross-sectional & Prevalence & 610 & 28 & NA & $4.59[3.07-6.57]$ \\
\hline Calza L, et al $^{16}$ & 2011 & Cross-sectional & Prevalence & 755 & 34 & NA & $4.50[3.14-6.24]$ \\
\hline Howard AA, et al ${ }^{17}$ & 2006 & Cross-sectional & Prevalence & 643 & 71 & NA & $11.04[8.73-13.72]$ \\
\hline Brown TT, et al ${ }^{18}$ & 2005 & Cohort(Index) & Prevalence & 568 & 68 & NA & $11.97[9.42-14.93]$ \\
\hline
\end{tabular}

In the Data collection on adverse effects of anti-HIV drugs (D: A: D) study conducted in 33, 389 people liv ing with HIV who were receiving antiretroviral treatment, with average age of 38 years and average Body Mass Index (BMI) of 23.0
$\mathrm{Kg} / \mathrm{m}^{2}$, followed over 130,151 person-years of follow-up from 11 cohorts and 212 clinics in Europe, US, Argentina and Australia, diabetes was diagnosed in 744 patients. The incidence rate of diabetes in this study was 5.72 per 1,000 
person-years of follow up.In this study, the incidence of Diabetes has increased with cumulative exposure to combination antiretroviral treatment. About three-fourth of the study subjects enrolled in to this study were men[8].

In the SwissHIV cohort study, 123 of 6,513 persons included in the follow up developed diabetes during 27,798 person-years of follow-up(mean duration of follow up was 4.3 years). This was equivalent to an incidence rate of 4.42 cases per 1000 person-years. This study has reported an increased incidence rate ratio among male study subjects and among those who use protease inhibitors and some nucleoside reverse transcriptase inhibitors (NRTI).The median age and the median BMI among the study subjects included in this cohort study were 38 years and $22.5 \mathrm{~kg} / \mathrm{m} 2$ respectively. Nearly $70 \%$ of the study subjects in this study were men[9].

In the Women's Interagency HIV study conducted in six cities of United States, Diabetes was diagnosed in 116 HIV-infected $(n=1524)$ and 36 HIV-uninfected $(n=564)$ wo men over 6, 802 person-years (4962 for HIV infected and 1840 for uninfected). The incidence of diabetes among HIV infected was about 2.33 cases per 100 person years. HIV-infected women who were not taking antiretroviral therapy had a diabetes incidence rate of 1.53 per 100 person-years. Women who were takinghighly active anti-retroviral treatment containing a protease inhibitor had a rate of 2.50 per 100 person-years and those reporting non-protease inhibitor-containing HAART had a rate of 2.89 per 100 person-years. The median age among the HIV positive women was 39.2 years while the median BMI was $26.8 \mathrm{~kg} / \mathrm{m}^{2}$. In this study, cumulative exposure to NRTI was associated with increased incidence of Diabetes[10].
In a cohort study of $1046 \mathrm{HIV}$-infected patients in 47 French clinical sites, representing 7,846 person-years of follow-up, 111 patients developed diabetes. The incidence rate was 14.1 per 1,000 person-years of follow up. The incidence rate in this study was 14.6 in men and 12.6 in wo men[11].

In another study conducted in Taiwan among 824 HIV-infected patients attended from 1993 to 2006 at National Taiwan University Hospital, 50 incident cases of Diabetes were diagnosed, resulting in an incidence of 13.1 cases per 1,000 person-years of follow-up. In this study, family history, exposure to zidivudine and current use of protease inhibitors were found to be the major risk factors for development of Diabetes[12]. The distribution of the incidence rates and their $95 \%$ confidence intervals are shown in the Figure 2.

\subsection{Prevalence of Diabetes Comorbi dity}

In the seven studies that have reported prevalence of diabetes, 41,068 people living with HIV were studied. Among these population, 1,724 prevalent cases of diabetes were identified. The reported prevalence rates ranged fro $\mathrm{m}$ $2.85 \%$ in the D:A: D baseline prevalence to $14.9 \%$ in the baseline prevalence of veterans ageing cohort study.

In the Data Collection on Adverse Events of Anti-HIV Drugs Study, 952 of the 33,389 patients had diagnosis of diabetes at index visit. This makes the bas eline prevalence of diabetes to be $2.85 \%$ [8].In the veterans ageing cohort study of 3,227 HIV-infected individuals, the bas eline prevalence of diabetes was $14.9 \%$ in the HIV infected as compared to $21.4 \%$ in the HIV unin fected group. Most of this difference was attributed to the differences in body mass index[13].

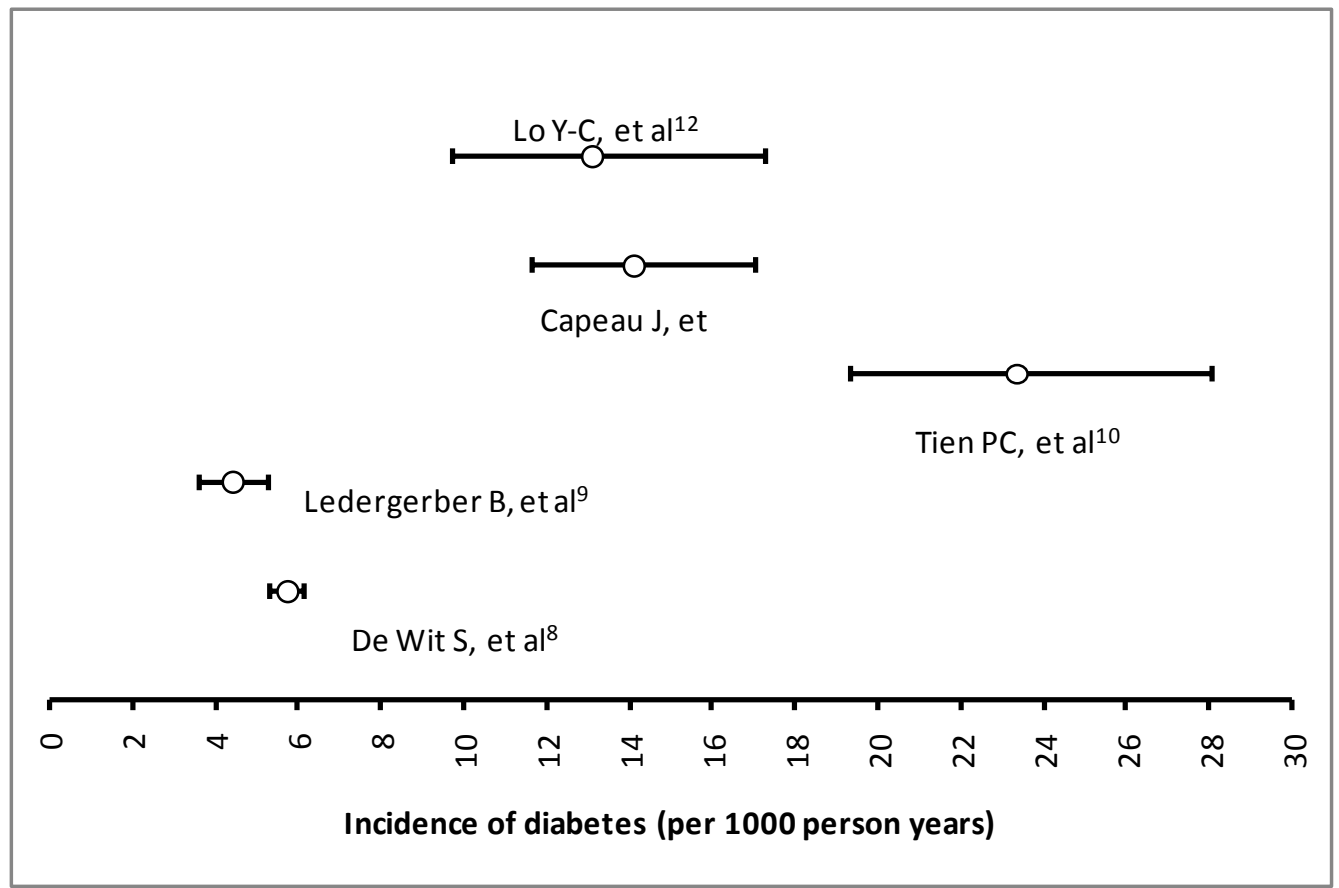

Figure 2. Diabetes incidence rates (per 1000 person-years) and their $95 \% \mathrm{Cl}$ 


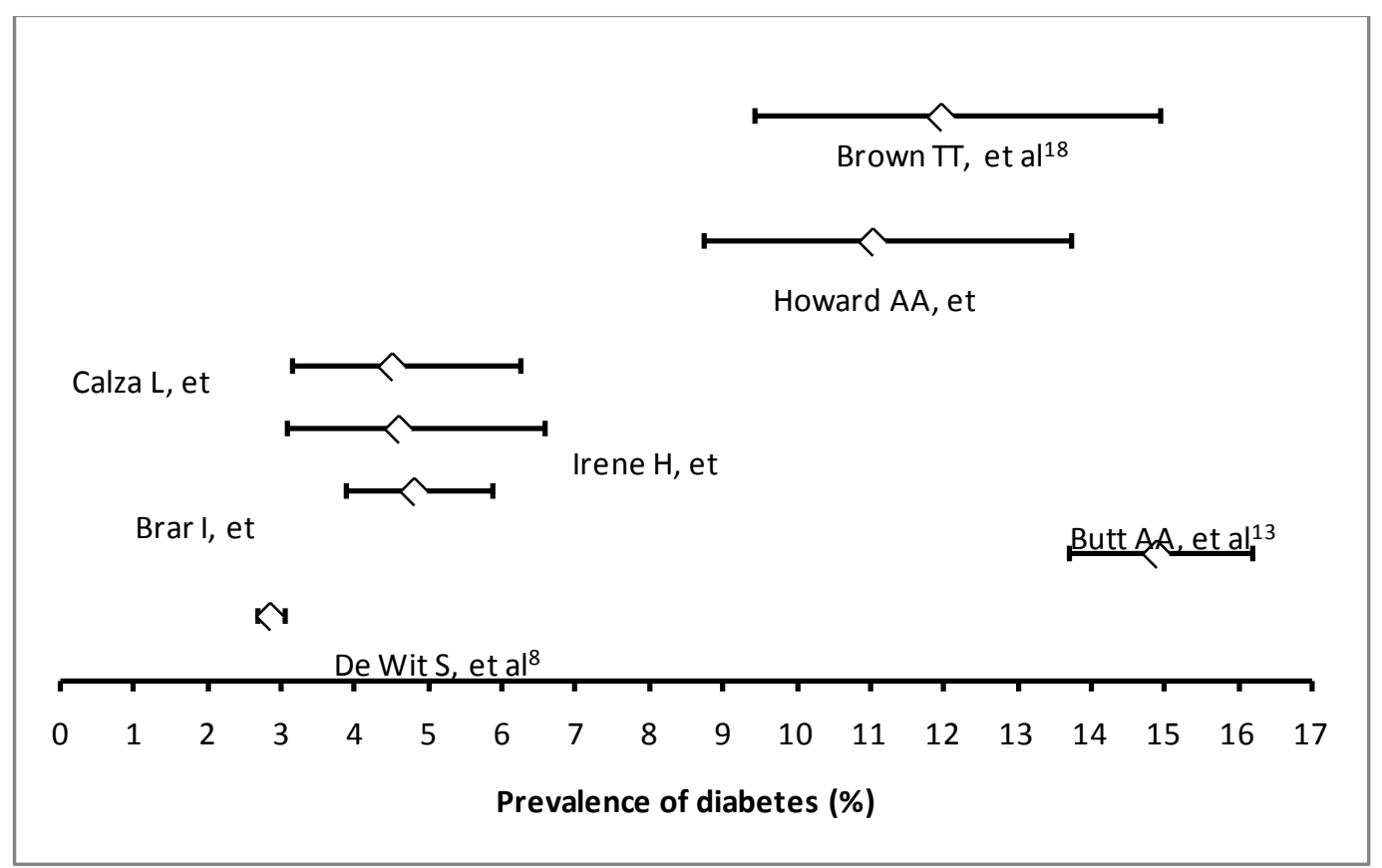

Figure 3. Reported prevalences (\%) of Diabetes among PLHIV

In a study of comparison of factors associated with prevalent diabetes mellitus among HIV-infected antiretroviral naive individuals enrolled in to Community Programs for Clinical Research on AIDS (CPCRA) clinical trials andadults enrolled in to the National Health and Nutritional Examination Survey (NHANES), the prevalence of self-reported Diabetes in the CPCRA clinical trials and the NHANES cohorts was $3.3 \%$ and $4.8 \%$, respectively[14].

In a study of the prevalence of diabetes among $610 \mathrm{HIV}$ positive asymptomatic patients in Botswana, the prevalence of diabetes was found to be $4.6 \%$ as compared to $7.2 \%$ for the general population in Botswana. A mong those above 40 years of age, the prevalence was $10.8 \%$. Older age and higher BMI were associated with higher diabetes prevalence in this study[15].

In a study conducted on 755 HIV positive individuals attending tertiary care hospital for routine clinical and laboratory follow-up between July and September 2009 in Italy, the prevalence of diabetes was found to be $4.5 \%$. A longer exposure to antiretroviral therapy and a diagnosis of lipodystrophy syndrome were significantly associated with higher levels of diabetes prevalence[16].

In a study of 643 older men, the prevalence of diabetes based on Oral Glucose Tolerance Test and among HIV infected anti-retroviral naïve individuals was $11 \%$ as compared to $8 \%$ in HIV uninfected individuals. In this study, age and Ethnicity were associated with abnormal Oral Glucose Tolerance Test (OGTT) results[17].

In the Multi-centre AIDS Cohort study, 68 (11.97\%) of the $568 \mathrm{HIV}$-infected men had prevalent Diabetesat the baseline visit. The prevalence of diabetes was $14 \%$ among those 411 HIV positive men who were using antiretroviral treatment. A mong $157 \mathrm{HIV}$ positive men who were not using highly active anti-retroviral treatment, 11 have diabetes and the prevalence of Diabetes at index visit among this group was about $7 \%[18]$.

\section{Discussion}

The incidence of Diabetes comorbidity among people living with HIV generally looks to be similar tothat of the general population. But this incidence has shown higher variability between studies with higher person years of follow up and those studies with re latively lowerperson years of follow up. Studies with higher person years of follow up seem to have a lower incidence of diabetes comorbidity than those with lower person years of follow up. The D: A: D study and the Swis s cohort study $[8,9]$ which have very high person years of follow up have reported incidence rates which are lower than all other reported values.

The prevalences of Diabetes comorbidity, which were outputs of cross-sectional studies in most cases, have also shown wider variability among the included studies. The prevalence of Diabetes in the respective general population, the socio-demographic profiles of the study subjects and duration on treatment may be the main factors related with this variability.

The relatively higher level of incidence and prevalence of diabetes among people living with HIV, and particularly among those who are receiving anti-retroviral treatment, warrants the screening of PLHIV for hyperglycaemia both at time of enrolment and during follow up period of HIV treatment. Whether this screening should be universally implemented for all people living with HIV or selectively for those with other risk factors needs further investigation. Screening during follow up periods while a person is on HIV treatment should also be the main stay in the prevention and control of diabetes among people living with HIV. Thus, 
diabetes screening programs need to be integrated with HIV treatment programs.

With regard to the limitations of this study, aggregation of the incidence and prevalence of diabetes comorbid ity among people living with HIV in to a single summary figure was not possible due to the heterogeneity of the included studies. This heterogeneity has also increased the variability among the reported incidence and prevalence rates. Another limitation of this study is that it was not possible to present the disaggregated incidence and prevalence rates for PLHIV taking ART and those not taking ART as only a few of studies have reported disaggregated values. Lastly, diabetes incidence and prevalence rates for the general population fro $m$ wh ich the study subjects were drawn were not available for comparison.

In conclusion, this review has summarized the incidence and prevalence of diabetes comorbid ity among people living with HIV. High variability in the reported incidence and prevalence rates were observed among the studies. Comparison of diabetes incidence and prevalence between HIV infected and uninfected people is also not consistent among studies. Diabetes prevalence in the general population, Sample sizes, duration of follow up and year of the study may be the possible factors associated with this variability. The study findings, in general, are not in favour of a significantly increased magnitude of diabetes comorbid ity a mong people living with HIV. However, given the lower average age of the study participants, regular monitoring and screening of blood sugar levels of people living with HIV are reco mmended.

Funding: None

Conflict of interest: None declared

Ethical approval: Not required

\section{REFERENCES}

[1] Young F,Critchley JA, Johnstone LK, Nigel C Unwin NC. A review of co-morbidity between infectious and chronic disease in Sub-Saharan Africa: TB and Diabetes Mellitus, HIV and Metabolic Syndrome, and the impact of globalization. Globalization and Health 2009, 5:9

[2] Stevenson CR, Forouhi NJ, Roglic G, Williams BG, Lauere JA, Dye C, et al. Diabetes and tuberculosis: the impact of the diabetes epidemic on tuberculosis incidence. BMC Public Health 2007, 7:234

[3] World Health Organization. Priority research questions for TB/HIV in HIV prevalent and Resource limited settings. 2010, Switzerland.

[4] Kalilani L, Atashili J. Measuring additive interaction using odds ratios. Epidemiologic perspectives and innovations 2006 , $3: 5$

[5] International Diabetes Federation. Diabetes in low-, middle-, and high income countries.Available at: http://www.idf. org/diabetesatlas/5e/diabetes-in-low-middle-and-high-incom e-countries Accessed on March 15, 2012.

[6] Misganaw A, Mariam DH, Araya T. The double mortality burden among adults in Addis Ababa, Ethiopia, 2006-2009. Prev Chronic Dis 2012;9:110142.

[7] Kalra S, Kalra B, Agrawal N, Unnikrishnan AG. Understanding diabetes in patients with HIV/AIDS. Diabetology \& Metabolic Syndrome 2011, 3:2

[8] De Wit S, Sabin CA, Weber R, Worm SW, Reiss P, Cazanave $\mathrm{C}$, et al. Incidence and risk factors for new-onset diabetes in HIV-infected patients: the data collection on adverse effects of anti-HIV drugs (D:A:D) study. Diabetes Care. 2008; 31(6):1224-1229

[9] Ledergerber B, Furrer H, Rickenbach M, Lehmann R, Elzi L, Hirschel B, et al. Factors associated with the incidence of type 2 diabetes mellitus in HIV-infected participants in the Swiss HIV Cohort Study. Clin Infect Dis. Jul 1 2007;45(1):111-119.

[10] Tien PC, Schneider MF, Cole SR, Levine AM, Cohen M, DeHovitz J, et al. Antiretroviral therapy exposure and incidence of diabetes mellitus in the Women's Interagency HIV Study. AIDS. 2007 Aug 20;21(13):1739-1745

[11] Capeau J, Bouteloup V, Katlama C, Bastard JP, Guiyedi V, Salmon-Ceron D, et al. Ten-year diabetes incidence in 1046 HIV-infected patients started on a combination antiretroviral treatment. AIDS. 2012 Jan 28;26(3):303-14.

[12] Lo YC, Chen MY, Sheng WH, Hsieh SM, Sun HY, Liu WC, et al. Risk factors for incident diabetes mellitus among HIV-infected patients receiving combination antiretroviral therapy in Taiwan: a case-control study. HIV Medicine (2009), 10, 302-309

[13] Butt AA, McGinnis K, Rodriguez-Barradas MC, Crystal S, Simberkoff M, Goetz MB, et al. HIV infection and the risk of diabetes mellitus. AIDS. 2009 Jun 19;23(10):1227-1234.).

[14] Brar I, Shuter J, Thomas A, Daniels E, Absalon J. A comparison of factors associated with prevalent diabetes mellitus among HIV-Infected antiretroviral-naive individuals versus individuals in the National Health and Nutritional Examination Survey cohort. J Acquir Immune DeficSyndr. 2007 May 1;45(1):66-71

[15] Irene H, Baum MK, Huffman F, BussmannH, Dusara P, Makhema J, et al. Diabetes in HIV Positive Adults in Botswana: Nutritional and Demographic Characteristics. FASEB J. April 200923 (Meeting Abstract Supplement) 547.15

[16] Calza L, Masetti G, Piergentili B, Trapani F, Cascavilla A, Manfredi R, et al. Prevalence of diabetes mellitus, hyperinsulinaemia and metabolic syndrome among 755 adult patients with HIV-1 infection. Int J STD AIDS January 2011 vol. 22 no. $143-45$

[17] Howard AA, Floris-Moore M, Lo Y, Arnesten JH, Fleischer $\mathrm{N}$,Klein RS. Abnormal glu cose metabolism among older men with or at risk of HIV infection. HIV Medicine (2006), 7, 389-396

[18] Brown TT, Coles SR, Li X, Kingsley LA, Palella FJ, Riddler SA, et al. Antiretroviral therapy and the prevalence and incidence of Diabetes mellitus in the multicenter AIDS cohort study. Arch Intern Med. 2005;165:1179-1184 